\title{
DIPTERA MUSCIDAE DO ESTADO DO RIO DE JANEIRO (BRASIL)
}

\author{
Márcia Souto Couri ${ }^{1,3}$ \& Cláudio Jose Barros de Carvalho ${ }^{2,3}$
}

Biota Neotropica v5 (n2) - http://www.biotaneotropica.org.br/v5n2/pt/abstract?inventory+BN01505022005

Recebido em 14/12/04

Versão reformulada recebida em: 01/05/05

Publicado em 03/08/2005

\footnotetext{
${ }^{1}$ Museu Nacional (http://acd.ufrj.br/museu/), Quinta da Boa Vista, São Cristóvão, 20940-040 Rio de Janeiro, RJ, Brasil e-mail: mcouri@attglobal.net

${ }^{3}$ Universidade Federal do Paraná (http://www.ufpr.br/), Caixa Postal 19020, 81531-990, Curitiba, Paraná, Brasil e-mail: cjbcarva@ufpr.br

${ }^{3}$ Bolsista de Produtividade em Pesquisa, CNPq
}

\begin{abstract}
A list of the species of Muscidae (Diptera) recorded in the State of Rio de Janeiro, Brazil is presented. A total of 843 species of 85 genera is known from the Neotropical Region. In the State of Rio de Janeiro, 154 species of 46 genera have been recorded.
\end{abstract}

Key words: Diptera, Muscidae, list of species, Rio de Janeiro.

\section{Resumo}

Uma lista de espécies de Muscidae (Diptera) assinaladas no Estado do Rio de Janeiro, Brasil é apresentada. Um total de 843 espécies de 85 gêneros é conhecido na Região Neotropical. No Estado do Rio de Janeiro, 154 espécies de 46 gêneros têm sido registradas.

Palavras-chave: Diptera, Muscidae, lista de espécies, Rio de Janeiro.

http://www.biotaneotropica.org.br 


\section{Introdução}

Os Muscidae são uma numerosa família de Diptera, com cerca de 4.500 espécies descritas e com ocorrência em todas as regiões biogeográficas. Destas, 843 espécies são reconhecidas na Região Neotropical (CARVALHO et al, 2005).

CARVALHO (1989), CARVALHO \& COURI(2002)e COURI \& CARVALHO (2002) apresentaram resenhas dos principais trabalhos sobre classificação de Muscidae no Brasil. Um resumo destas informações acrescido de comentários segue abaixo.

Do final do século passado ao início deste, contribuições para a classificação dos Muscidae podem ser encontradas em SCHINNER (1868); BRAUER \& BERGENSTAMM(1889)eSCHNABL\&DZIEDZICKI(1911).

Até 1920 , os múscidas eram considerados pertencentes à família Anthomyiidae, caracterizada pelo mero nu, nervura $M_{1+2}$ reta ou quase reta no terço apical, ausência de coloração metálica, entre outros caracteres. De acordo com esta conceituação, os Anthomyiidae incluíam os Anthomyiinae, Coenosiinae, Lispinae, Limnophorinae, Mydaeinae, Fanniinae e Phaoninae.

Depois de 1920, uma das principais contribuições para a classificação dos Muscidae, foi apresentada por MALLOCH (1934). Este autor considerou como Muscidae todas as espécies de Diptera Calyptratae sem cerdas desenvolvidas no mero, incluindo Scatophaginae e Egininae. Ele dividiu esta família em 7 sub-famílias: Anthomyiinae, Fanniinae, Coenosiinae, Lispinae, Phaoninae, Stomoxyinae e Muscinae.

SÉGUY (1937) considerou como Muscidae todas as espécies sem cerdas fortes no mero, dividindo-os em 15 sub-famílias.

Entre as várias classificações de Muscidae propostas, com base principalmente nos caracteres dos adultos (e.g. HENNIG, 1956-1964b), a primeira baseada na metodologia cladística foi proposta por HENNIG (1965). PONT (1972, 1977, 1986a) seguiu esta classificação com poucas modificações.

SKIDMORE (1985) estudou 440 espécies e propôs uma classificação baseada principalmente em caracteres das formas imaturas.

CARVALHO (1989) propôs uma classificação com base em análise cladística, que incluiu 27 gêneros, 12 deles exclusivamente neotropicais. A classificação resultante foi muito similar a apresentada por SKIDMORE (1985). Foram reconhecidas 7 sub-famílias (6 neotropicais) e 8 tribos: Acanthipterinae, Atherigoninae, Muscinae (Stomoxini e Muscini), Azelinae (Azeliini e Reinwardtiini) Phaoninae, Mydaeinae (Graphomyini e Mudaeini) e Coenosiinae (Limnophorini e Coenosiini).

CARVALHO\& COURI(2002)eCOURI \& CARVALHO (2002) apresentaram uma revisão taxonômica dos Muscidae da Região Neotropical, com chaves de identificação para gêneros e espécies, diagnoses de sub-famílias, tribos e gêneros e discussões sobre as relações supra-genéricas.
A classificação aqui seguida é a mesma de CARVALHO et al (2005), que se refere a um catálogo dos Muscidae neotropicais.

\section{Morfologia e Biologia}

Os adultos podem ser reconhecidos pela ausência da série vertical de cerdas no mero, pela presença de calíptras, fêmeas com menos de 7 pares de espiráculos (6, na maioria dos gêneros) no abdomen e machos sem o par de glândulas acessórias do sistema reprodutor.

Os ovos são ovais-alongados com duas pregas longitudinais dorsais, ao longo das quais o córion se rompe quando a larva eclode. As larvas são sub-cilíndricas ou pouco achatadas, afilando anteriormente, apresentam espessamentos cuticulares e espículas. (SKIDMORE 1985).

Os adultos podem ser predadores, hematófagos, saprófagos ou necrófagos. As larvas ocupam habitats extremamente variados, tais como esterco de mamíferos e carne putrefata, matéria orgânica vegetal e animal em decomposição, madeira, fungos, ninhos e tocas de mamíferos, entre outros. As espécies com larvas saprófagas e coprófagas podem ser benéficas, contribuindo na reciclagem de desperdícios orgânicos, enquanto as larvas carnívoras podem se alimentar de larvas de espécies sinantrópicas, desempenhando o papel de reguladores destas populações (CARVALHO et. al, 1993).

Muitas espécies estão associadas ao homem e aos animais domésticos. Apresentam grande importância econômica e considerável importância médica, principalmente como vetores mecânicos de agentes causadores de várias doenças. Algumas espécies são ainda causadoras de miíases obrigatórias ou facultativas (GREENBERG, 1971; GUIMARÃES \& PAPAVERO, 1999)

\section{Os Muscidae na Região Neotropical}

O conhecimento taxonômico dos Muscidae na região Neotropical está reunido em CARVALHO \& COURI (2002)e COURI \& CARVALHO (2002), que apresentam uma chave para os gêneros da Região Neotropical, diagnoses das subfamílias, tribos e gêneros e chaves para identificação das espécies com ocorrência nesta região.

A Tabela 1 (CARVALHO \& COURI, 2002 e COURI \& CARVALHO, 2002, modificadas com base em CARVALHO et al (2005) mostra os gêneros e o número de espécies de Muscidae no Rio de Janeiro, no Brasil e na Região Neotropical.

As abreviaturas utilizadas para as instituições onde estão localizados o material tipo são: AMNH (American Museum of Natural History, Nova Iorque, EUA); BMNH (The Natural History Museum, London, Reino Unido); CNC (Canadian National Collection, Ottawa, Canadá); IMLA (Universidad Nacional de Tucuman, Fundación e Instituto Miguel Lillo, Argentina, Tucuman,); FIOC (Fundação 
Tabela 1. Sub-famílias, Tribos e Gêneros de Muscidae, incluindo o número de espécies no Brasil, e Região Neotropical

\begin{tabular}{|c|c|c|c|c|c|}
\hline .Sub-família & Tribo & Gênero & Rio de Janeiro & Brasil & $\begin{array}{l}\text { Região } \\
\text { Neotropical }\end{array}$ \\
\hline Atherigoninae & & Atherigona & 1 & 1 & 1 \\
\hline \multirow{15}{*}{ Muscinae } & \multirow[t]{12}{*}{ Muscini } & Archaeopolietes & 0 & 0 & 1 \\
\hline & & Biopyrellia & 1 & 1 & 1 \\
\hline & & Cyacyrtoneura & 0 & 1 & 1 \\
\hline & & Morellia & 6 & 14 & 18 \\
\hline & & Musca & 1 & 1 & 2 \\
\hline & & Neomyia & 0 & 1 & 1 \\
\hline & & Neorypellia & 0 & 1 & 1 \\
\hline & & Parapyrellia & 1 & 2 & 2 \\
\hline & & Polietina & 6 & 13 & 18 \\
\hline & & Trichomorellia & 2 & 2 & 9 \\
\hline & & Sarcopromusca & 1 & 2 & 2 \\
\hline & & Xenomorellia & 2 & 2 & 2 \\
\hline & \multirow[t]{3}{*}{ Stomoxyini } & Haematobia & 1 & 1 & 1 \\
\hline & & Neivamyia & 0 & 3 & 4 \\
\hline & & Stomoxys & 1 & 1 & 1 \\
\hline \multirow[t]{16}{*}{ Azeliinae } & \multirow[t]{7}{*}{ Azeliini } & Azelia & 0 & 0 & 1 \\
\hline & & Drymeia & 0 & 0 & 1 \\
\hline & & Hydrotaea & 1 & 1 & 8 \\
\hline & & Micropotamia & 2 & 3 & 4 \\
\hline & & Ophyra & 4 & 4 & 7 \\
\hline & & Potamia & 0 & 0 & 2 \\
\hline & & Thricops & 0 & 0 & 1 \\
\hline & \multirow[t]{9}{*}{ Reinwardtiini } & Brachygasterina & 0 & 0 & 4 \\
\hline & & Chaetagenia & 0 & 1 & 1 \\
\hline & & Correntosia & 0 & 1 & 2 \\
\hline & & Dalcyella & 0 & 1 & 1 \\
\hline & & Itatingamyia & 0 & 1 & 1 \\
\hline & & Muscina & 1 & 1 & 4 \\
\hline & & Palpibracus & 0 & 0 & 17 \\
\hline & & Philornis & 10 & 29 & 49 \\
\hline & & Psilochaeta & 1 & 3 & 5 \\
\hline
\end{tabular}

http://www.biotaneotropica.org.br 


\begin{tabular}{|c|c|c|c|c|c|}
\hline & & Reinwardtia & 0 & 1 & 1 \\
\hline & & Synthesiomyia & 1 & 1 & 1 \\
\hline \multirow[t]{11}{*}{ Cyrtoneurininae } & & Arthurella & 0 & 0 & 1 \\
\hline & & Cariocamyia & 2 & 2 & 2 \\
\hline & & Charadrella & 1 & 2 & 3 \\
\hline & & Chortinus & 0 & 1 & 2 \\
\hline & & Cyrtoneurina & 3 & 7 & 10 \\
\hline & & Cyrtoneuropsis & 9 & 25 & 33 \\
\hline & & Mulfordia & 0 & 0 & 3 \\
\hline & & Neomuscina & 13 & 16 & 38 \\
\hline & & Neomusciniopsis & 0 & 1 & 1 \\
\hline & & Neurotrixa & 0 & 1 & 1 \\
\hline & & Pseudoptilolepis & 2 & 6 & 6 \\
\hline \multirow{4}{*}{\multicolumn{2}{|c|}{ Phaoniinae }} & Dolichophaonia & 5 & 15 & 27 \\
\hline & & Helina & 6 & 23 & 90 \\
\hline & & Phaonia & 6 & 20 & 46 \\
\hline & & Souzalopesmyia & 1 & 3 & 5 \\
\hline \multirow{7}{*}{\multicolumn{2}{|c|}{ Mydaeinae }} & Brontaea & 3 & 4 & 4 \\
\hline & & Graphomya & 2 & 3 & 12 \\
\hline & & Hemichlora & 1 & 1 & 1 \\
\hline & & Mydaea & 2 & 4 & 20 \\
\hline & & Myospila & 3 & 4 & 6 \\
\hline & & Scenetes & 0 & 0 & 1 \\
\hline & & Scutellomusca & 0 & 1 & 1 \\
\hline \multirow[t]{12}{*}{ Coenosiinae } & Limnophorini & Agenamyia & 1 & 1 & 2 \\
\hline & & Albertinella & 0 & 1 & 1 \\
\hline & & Drepanocnemis & 0 & 0 & 3 \\
\hline & & Limnophora & 7 & 21 & 43 \\
\hline & & Lispe & 2 & 5 & 12 \\
\hline & & Lispoides & 1 & 1 & 19 \\
\hline & & Pachyceramyia & 1 & 1 & 4 \\
\hline & & Rhabdotoptera & 0 & 0 & 1 \\
\hline & & Spilogona & 1 & 1 & 8 \\
\hline & & Syllimnophora & 0 & 2 & 28 \\
\hline & & Tetramerinx & 0 & 0 & 2 \\
\hline & & Thaumasiochaeta & 0 & 0 & 7 \\
\hline
\end{tabular}




\begin{tabular}{|c|c|c|c|c|c|}
\hline \multirow{16}{*}{\multicolumn{2}{|c|}{ Coenosiini }} & Apsil & 0 & 0 & 10 \\
\hline & & Bithoracochaeta & 4 & 7 & 10 \\
\hline & & Coenosia & 9 & 12 & 40 \\
\hline & & Cordiluroides & 2 & 3 & 6 \\
\hline & & Insulamyia & 1 & 1 & 1 \\
\hline & & Neodexiopsis & 21 & 29 & 87 \\
\hline & & Notoschoenomyza & 0 & 0 & 7 \\
\hline & & Oxytonocera & 0 & 1 & 1 \\
\hline & & Pentacricia & 0 & 0 & 1 \\
\hline & & Pilispina & 1 & 6 & 6 \\
\hline & & Plumispina & 1 & 1 & 1 \\
\hline & & Reynoldsia & 0 & 0 & 9 \\
\hline & & Schoenomyza & 0 & 0 & 18 \\
\hline & & Schoenomyzina & 0 & 0 & 6 \\
\hline & & Spathipheromyia & 0 & 0 & 14 \\
\hline & & Stomopogon & 0 & 0 & 6 \\
\hline Total & & 85 gêneros & 154 & 325 & 843 \\
\hline
\end{tabular}

Instituto Oswaldo Cruz, Rio de Janeiro, Brasil); HNHM (Hungarian Natural History Museum, Hungria, Budapeste); LSUK (Linnean Society, United Kingdom, Londres,); MCZ (Harward University, Museum of Comparative Zoology, Cambridge, Massachussetts, EUA); MNHN (Muséum National d'Histoire Naturelle, Paris, França); MNRJ (Museu Nacional, Rio de Janeiro, Brasil); NMW (Naturhistorisches Museum, Viena, Áustria); NHRS (Naturhistoriska Riksmuseet, Estocolmo, Suécia); RMNH (Nationaal Natuurhistorische Museum ("Naturalis”) [antigo Rijksmuseum van Natuurlijke Historie], Holanda, Leiden]; SMF (Forschungsinstitut und Naturmuseum Senckenberg, Alemanha, Frankfurt); SMT (Staaliches Museum für Tierkunde, Drenden, Alemanha); SEMC (Lawrence, University of Kansas, Snow Entomological Museum, EUA, Kansas); USNM (National Museum of Natural History, Washington, DC, EUA); ZMAN (Universiteit van Amsterdam, Instituut voor Taxonomische Zoologie, Zoologisch Museum, Holanda, Amsterdam); ZMUC (Universitetets Zoologisk Museum, Copenhage, Dinamarca); ZMHB (Museum für Naturkunde, HumboldtUniversität, Berlim, Alemanha).
Muscidae do Rio de Janeiro

Subfamília Atherigoninae Fan, 1965

Gênero Atherigona Rondani

Atherigona Rondani, 1856:97. Espécie-tipo, Anthomyia varia Meigen (des. orig.).

orientalis Schiner, 1868:295. Holótipo fêmea, NMW [PONT, 1986b:18]. Localidade-tipo: Ilhas Nicobar, Tellnschong. Distr. - Argentina, Barbados, Brasil, Colombia, Cuba, El Salvador, Equador, Galápagos, Guiana, Ilhas Virgens, Jamaica, México, Panamá, Paraguai, Peru, Puerto Rico, Republica Dominicana, Santo Domingo, Santa Lucia, St. Kitts, Trinidade, Venezuela.

Subfamília Muscinae Latreille, 1802

Tribo Muscini Latreille, 1802 
Gênero Biopyrellia Townsend

Biopyrellia Townsend, 1932:105. Espécie-tipo, Musca bipuncta Wiedemann (des. orig.).

bipuncta Wiedemann, 1830:417 (Musca). Lectótipo fêmea, NMW, paralectótipos NMW/SMF [des. PONT, 1997]. Localidade-tipo: “Brasil”. Distr. Argentina, Bolívia, Brasil, Costa Rica, México, Panamá, Paraguai, Trinidad, Venezuela.

\section{Gênero Morellia Robineau Desvoidy}

Morellia Robineau Desvoidy, 1830:405. Espécie-tipo, agilis Robineau Desvoidy (TOWNSEND, 1916:8) = hortorum (Fallén).

concacata Pamplona, 1986:653, figs. 6 12. Holótipo macho, MNRJ, parátipos MNRJ. Localidade-tipo: Brasil, Rio de Janeiro, Ilha do Governador. Distr. Brasil.

couriae Pamplona, 1986:656, figs. 13 19. Holótipo macho, MNRJ, parátipos MNRJ. Localidade-tipo: Brasil, Rio de Janeiro, Petrópolis, Retiro. Distr. Brasil.

humeralis Stein, 1918:204 (Pyrellia). Síntipos macho/ fêmea, NMW/SMT/ZMHU [destruídos HNHM]. Localidade-tipo: Brasil, Teresópolis [Rio de Janeiro], Espirito Santo; Peru; Bolívia. Distr. Bolívia, Brasil, Peru.

nigricosta Hough, 1900:216, figs. 7 e 8. Síntipos macho e fêmea, SEMC. Localidade-tipo: Brasil, Mato Grosso, Chapada dos Guimarães. Distr. Brasil, Costa Rica, Guiana, Paraguai, Peru.

ochricornis Wiedemann, 1830:408 (Musca). Lectótipo macho, NMW [des. PONT, 1997]. Localidade-tipo: "Brasil”. Distr. Brasil, Cuba, Galápagos, México, Puerto Rico.

violacea Robineau Desvoidy, 1830:463 (Pyrellia). Síntipos ?sexo, destruídos. Localidade-tipo: “Brasil”. DistrArgentina, Brasil, Colombia, Costa Rica, Ecuador, Jamaica, México, Peru, Paraguai, Venezuela.

\section{Gênero Musca Linnaeus}

Musca Linnaeus, 1758:589. Espécie-tipo, domestica Linnaeus (I.C.Z.N. 1925, Opinião 82).

domestica Linnaeus, 1758:596. Síntipos ?sexo, destruídos [não em LSL, veja PONT, 1981:168]. Localidadetipo: Europa e America. Distr. Argentina, Barbados, Belize, Bolivia, Brasil, Chile, Colombia, Costa Rica, Cuba, Equador, Galápagos, Guatemala, Granada, Guiana, Haiti, Ilha da Páscoa, Ilhas Guadalupe, Ilhas Juan Fernández, Ilhas Mona, Ilhas Virgens, Jamaica, México, Nassau, Nicarágua, Panamá, Paraguai, Peru, Puerto Rico, Republica Dominicana, San Salvador, Santa Lucia, Sao Vincente, Surinam, Trinidad, Uruguai, Venezuela, cosmopolita.

\section{Gênero Parapyrellia Townsend}

Parapyrellia Townsend, 1915:97. Espécie-tipo, Musca violacea Fabricius de autores (des. orig.) = maculipennis Macquart. maculipennis Macquart, 1846a:327, pr.17, fig.6; 1846b:199, pr.17, fig.6 (Pyrellia). Lectótipo fêmea, BMNH. Localidade-tipo: “Colombia e Brasil”. Distr. Bolivia, Brasil, Colombia, Cuba, Guiana, Ilha Guadalupe, Jamaica, México, Paraguai, Peru, Puerto Rico, Republica Dominicana, Trinidad, Venezuela.

\section{Gênero Polietina Schnabl \& Dziedzicki}

Polietina Schnabl \& Dziedzicki, 1911:218. Espécie-tipo, Aricia pruinosa Macquart (como pruinosus Bigot)(des. orig.).

bicolor Albuquerque, 1956e:10, figs. 24-33. Holótipo macho, MNRJ, Parátipos MNRJ. Localidade-tipo: Brasil, Rio de Janeiro, Tinguá. Distr. Brasil.

minor Albuquerque, 1956e:7, figs. 12-23. Holótipo macho, MNRJ. Localidade-tipo: Brasil, Rio de Janeiro, Angra dos Reis, Fazenda Japuíba. Distr. Brasil.

nigra Couri \& Carvalho, 1996: 825-828, figs 1-12. Holótipo macho, MNRJ, parátipo MNRJ. Localidade-tipo: Brasil, Rio de Janeiro. Distr.- Brasil.

orbitalis Stein, 1904:427 (Aricia). Holótipo fêmea, destruído [anteriormente HNMN]. Localidade-tipo: Peru, Vilcanota. Distr. Argentina, Bolivia, Brasil Peru.

prima Couri \& Machado, 1990:659, figs. 1-4. Holótipo fêmea, MNRJ, Parátipos MNRJ. Localidade-tipo: Brasil, Rio de Janeiro, Parque Nacional da Tijuca. Distr.- Brasil.

stellata Couri, 1982b:51, figs. 26 29. Holótipo macho, MNRJ. Localidade-tipo: Brasil, Rio de Janeiro, Represa Rio Grande. Distr. Brasil.

\section{Genus Trichomorellia Stein}

Trichomorellia Stein, 1918:204 (and 1919:109). Type species, Curtonevra cyanea sensu Stein, 1918 (mon.)..

flavipalpis Pamplona, 1983:4, figs. 78 (Dasymorellia). Holotype male, MNRJ. Type locality: Brazil, Rio de Janeiro, Itatiaia. Distr. Brazil.

trichops Malloch, 1923:527 (Dasymorellia). Holotype male, USMN, paratypes BMNH. Type locality: Peru, Ollantaybamba and Paltaybamba. Distr. Colombia, Ecuador, Peru, Bolivia, Brazil, Argentina.

\section{Gênero Sarcopromusca Townsend}

Sarcopromusca Townsend, 1927:209. Espécie-tipo, arcuata Townsend (mon.) = pruna (Shannon e Del Ponte).

pruna Shannon \& Del Ponte, 1926:581 (Orthellia). Holótipo fêmea, USMN. Localidade-tipo: Argentina, Jujuy, Calilegua. Distr. Argentina, Brasil, Costa Rica, Guatemala, Nicarágua.

Gênero Xenomorellia Malloch 
Xenomorellia Malloch, 1923:524. Espécie-tipo, holti Malloch (des. orig.).

holti Malloch, 1923:525. Holótipo macho, USNM, parátipo BMNH. Localidade-tipo: Peru [Brasil?], Alto Itatiaia, Serra do Itatiaia. Distr. Brasil, Peru.

montanhesa Albuquerque, 1952:2, figs. 1 13. Holótipo macho, MNRJ. Localidade-tipo: Brasil, Rio de Janeiro, Itatiaia, Macieira. Distr. Brasil.

Tribo Stomoxyini Meigen, 1824

\section{Gênero Haematobia Le Peletier \& Serville}

Haematobia Le Peletier \& Serville in LATREILLE et al, 1828:499 (como sub-gênero de Stomoxys Gedefroy). Espécie-tipo, Conops irritans Linnaeus (WESTWOOD, 1840:140, e I.C.Z.N. 1974, Opinão 1008).

irritans Linnaeus, 1758:604 (Conops). Lectótipo macho, LSUK [des. PONT, 1981:169]. Localidade-tipo: Suécia. Distr. África do Norte, América do Norte, Argentina, Ásia, Brasil Chile, Colombia, Costa Rica, Cuba, El Salvador, Europa, Guatemala, Guiana, Haiti, Havai, Ilhas Virgens, Jamaica, México, Nicarágua, Panamá, Puerto Rico, Sao Domingo, Venezuela.

\section{Gênero Stomoxys Gedefroy}

Stomoxys Gedefroy, 1762:449 e 538. Espécie-tipo, Conops calcitrans Linnaeus (I.C.Z.N. 1957, Opinião 441).

calcitrans Linnaeus, 1758:604 (Conops). Lectótipo fêmea, LSUK [des. PONT, 1981:168]. Localidade-tipo: Suécia. Distr. Argentina, Bahamas, Barbados, Belize, Bolivia, Brasil, Chile, Colombia, Costa Rica, Cuba, Equador, Galápagos, Granada, Guatemala, Guiana, Haiti, Ilha da Páscoa, Jamaica, México, Nicarágua, Panamá, Puerto Rico, Peru, Republica Dominicana, Sao Vincente, Tobago, Trinidade, Uruguai, Venezuela, cosmopolita.

Subfamilia Azeliinae Robineau Desvoidy, 1830

Tribo Azeliini Robineau Desvoidy, 1830

Gênero Hydrotaea Robineau Desvoidy.

Hydrotaea Robineau Desvoidy, 1830:509. Espécietipo, Musca meteorica Linnaeus (CURTIS, 1839:768).

nicholsoni Curran, 1939:2. Holótipo macho, AMNH. Localidade-tipo: Peru, Arequipa. Distr. Peru, Bolivia, Brasil, Chile, Argentina.

\section{Gênero Micropotamia Carvalho}

Micropotamia Carvalho, 1993b: 242. Espécie-tipo, Phaonia minuscula Albuquerque, 1955c (des. orig.). cilitibia Albuquerque, 1955c:375, figs. 813. (Phaonia). Holótipo macho, MNRJ. Localidade-tipo: Brasil, Mato Grosso, Salobra. Distr. Brasil.

minuscula Albuquerque, 1955c:373, figs. 17 (Phaonia). Holótipo macho, MNRJ. Localidade-tipo: Brasil, Rio de Janeiro, Itatiaia, Lago Azul. Distr. Brasil.

\section{Gênero Ophyra Robineau Desvoidy}

Ophyra Robineau Desvoidy, 1830:516. Espécie-tipo, nitida Robineau Desvoidy (RONDANI, 1866:70, 84) = ignava Harris.

aenescens Wiedemann, 1830:435 (Anthomyia). Lectótipo macho, NMW [des. PONT, 1997], paralectótipo NMW. Localidade-tipo: EUA, New Orleans. Distr. América do Norte, Argentina, Bolivia, Brasil, Chile, Cuba, Equador, Galápagos, Guiana, Ilha da Páscoa, Jamaica, México, Nicarágua, oeste da Região Paleártica, Pacífico Leste, Peru, Puerto Rico, Sao Vincente, Trinidade, Tobago, Uruguai, Venezuela.

albuquerquei Lopes, 1985:117, figs. 1 8. Holótipo macho, MNRJ, Parátipos MNRJ. Localidade-tipo: Brasil, Rio de Janeiro, Le Vallon, Alto da Mosela. Distr. Brasil.

chalcogaster Wiedemann, 1824:52 (Anthomyia). Lectótipo macho, UZMC [des. PONT, 1973:242]. Localidadetipo: Java. Distr. Afrotropical, Australásia, Brasil, Chile; Regiões Neártcia (Bermuda) e Oriental.

solitaria Albuquerque, 1958d:7, figs. 12 16. Holótipo fêmea, MNRJ. Localidade-tipo: Brasil (Goiás, Anápolis). Distr. Brasil.

Tribo Reinwardtiini Brauer \& Bergenstamm, 1889

Gênero Muscina Robineau Desvoidy

Muscina Robineau Desvoidy, 1830:406. Espécie-tipo, Musca stabulans Fallén (COQUILLETT, 1910:571).

stabulans Fallén, 1817:252 (Musca). Lectótipo macho, NHRS [des. PONT, 1984:294]. Localidade-tipo: Suécia. Distr. Argentina, Brasil, Chile, Costa Rica, Guatemala, México, Peru, Uruguai, Venezuela; cosmopolita.

\section{Gênero Philornis Meinert}

Philornis Meinert, 1890:315. Espécie-tipo, molesta Meinert (mon).

cinnamomina Stein, 1918:214 (Mydaea). Holótipo macho, destruído [anteriormente HNHM]. Localidadetipo: "Paraguai”. Distr. Brasil, Paraguai, Puerto Rico, Trinidad, Venezuela.

downsi Dodge \& Aitken, 1968:138, figs. 15. Holótipo macho, USNM, Parátipos BMNH/FIOC. Localidade-tipo: Trinidad, Cunaripo R. C. School. Distr. Brasil, Galapágos, Trindade. 
grandis Couri, 1984:481, figs. 20-21, 24 28. Holótipo macho, CNC?, Parátipos CNC?/MNRJ. Localidade-tipo: Equador, Pastaza, Pompeya, Rio Napo. Distr. Brasil, Equador, México.

insularis Couri, 1983:302, figs. 19 28. Holótipo macho, MNRJ. Localidade-tipo: Brasil, Rio de Janeiro, Ilha Grande. Distr. Brasil.

lopesi Couri, 1983:306, figs. 30 31. Holótipo fêmea, MNRJ. Localidade-tipo: Brasil, Rio de Janeiro, Angra dos Reis. Distr. Brasil.

sabroskyi Albuquerque, 1957c:11; nom.n. para blanchardi Albuquerque. Distr. Brasil, Guiana. setinervis Dodge, 1963:246. Holótipo fêmea, USNM. Localidade-tipo: Brasil, Rio de Janeiro. Distr. Brasil. torquans Nielsen, 1913:252, fig. 3 (Mydaea). Lectótipo macho, UZMC [des. DODGE, 1968:156]. Localidade-tipo: Argentina. Distr. Argentina, Brasil.

univittata Dodge, 1968:161. Holótipo fêmea, USNM. Localidade-tipo: Brasil, Rio de Janeiro. Distr. Brasil. vulgaris Couri, 1984:488, figs. 34, 35. Holótipo fêmea, CNC?, paratipo CNC? Localidade-tipo: Brasil, Amazonas, Manaus, Reserva Ducke. Distr. Brasil.

\section{Gênero Psilochaeta Stein}

Psilochaeta Stein, 1911:72. Espécie-tipo. Limnophora chlorogaster Bigot (orig. des.) = chlorogaster Wiedemann.

pampiana Shannon \& Del Ponte, 1926:577 (Phyronota). Holótipo macho, USNM. Localidade-tipo: Argentina, Tucumán. Distr. Argentina, Brasil.

\section{Gênero Synthesiomyia Brauer \& Bergenstamm}

Synthesiomyia Brauer \& Bergenstamm, 1893:96, 110 e 178. Espécie-tipo, brasiliana Brauer \& Bergenstamm (des. orig.) = nudiseta Wulp.

nudiseta Wulp, 1883:42 (Cyrtoneura). Holótipo fêmea, perdido [não BMNH/ZMAN/RNH]. Localidade-tipo: Argentina. Distr. Argentina, Bolivia, Brasil, Chile, Costa Rica, Cuba, Equador, Galápagos, Guiana, Ilha da Páscoa, Ilhas Virgens, Jamaica, México, Nicarágua, Panamá, Paraguai, Peru, Porto Rico, Sao Domingo, Trindade, Venezuela; tropicopolitana.

\section{Subfamilia Cyrtoneurininae Snyder, 1954}

\section{Gênero Cariocamyia Snyder}

Cariocamyia Snyder, 1951:1. Espécie-tipo, maculosa Snyder (orig. des.).

maculosa Snyder, 1951:1, fig. 1. Holótipo macho, AMNH. Localidade-tipo: Brasil, Guanabara, Rio de Janeiro, Grajaú. Distr. Brasil, Colombia. vibrissata Stein, 1918:222 (Mydaea). Holótipo fêmea, NMW. Localidade-tipo: Brasil, São Paulo, Iguape. Distr. Brasil.

\section{Gênero Charadrella Wulp}

Charadrella Wulp, 1896b:341. Espécie-tipo, macrosoma Wulp (mon.).

malacophaga Lopes, 1938:927, figs. 13 . Holótipo macho, FIOC. Localidade-tipo: Brasil, Rio de Janeiro. Distr. Brasil.

\section{Gênero Cyrtoneurina Giglio Tos}

Cyrtoneurina Giglio Tos, 1893:5. Espécie-tipo, uber Giglio Tos (COQUILLETT, 1910:530

alifusca Couri, 1982b:39, figs. 1 3. Holótipo fêmea, MNRJ. Localidade-tipo: Brasil, Rio de Janeiro, Tinguá. Distr. Brasil.

biseta Snyder, 1954:436. Holótipo macho, USNM. Localidade-tipo: Brasil, Rio de Janeiro. Distr. Brasil.

confusa Snyder, 1954:437. Holótipo macho, AMNH, Parátipos AMNH. Localidade-tipo: Panamá, Patilla Point. Distr. Brasil, Costa Rica, Panamá.

\section{Gênero Cyrtoneuropsis Malloch}

Cyrtoneuropsis Malloch, 1925:91 (como sub-gênero de Cyrtoneurina). Espécie-tipo, Spilogaster veniseta Stein (orig. des.).

fuscisquama Snyder, 1954:450 (Cyrtoneurina). Holótipo macho, USNM, Parátipos AMNH. Localidade-tipo: Brasil, Rio de Janeiro. Distr. Brasil.

incognita Snyder, 1954:452, fig. 17 (Cyrtoneurina). Holótipo macho, USNM, Parátipos USNM/AMNH. Localidade-tipo: Brasil (Rio de Janeiro). Distr. Brasil.

polystigma Wulp, 1896a : prancha 7, fig. 20 [\& 1896b:309] (Clinopera). Lectótipo macho, ZMAN [des. SNYDER, 1954:452]. Localidade-tipo: México, Medellin. Distr. Brasil, Costa Rica, El Salvador, Guatemala, México, Panamá, Paraguai.

praenubila Snyder, 1954:444, figs. 8, 9, 26, 37 e 53 (Cyrtoneurina). Holótipo macho, AMNH, Parátipos AMNH. Localidade-tipo: Trinidad, Port de Spain. Distr. Brasil, Granada, Panamá, Peru, Trinidade.

rescita Walker, 1861:315 (Aricia). Holótipo fêmea, BMNH. Localidade-tipo: México. Distr. Belize, Bolivia, Brasil, Colombia, Costa Rica, Cuba, Equador, Guatemala, Guiana, Ilha Sao Vicente, México, Nicarágua, Panamá, Paraguai, Peru, Porto Rico, Trindade, Venezuela.

seriata Stein, 1911:91 (Mydaea). Síntipos macho/ fêmea, SMT. Localidade-tipo: Peru, Vale de Lares e Rio Urubamba. Distr. Brasil, Colombia, Peru. 
spiloptera Wiedemann, 1830:433 (Anthomyia). Lectótipo fêmea, NMW [des. PONT, 1997]. Localidadetipo: "Brasil”. Distr. Belize, Bolivia, Brasil, Equador Panamá, Peru, Surinam.

varicolor Hough, 1900:226, pl. 45 figs. 14 e 15 (Muscina). Síntipos macho/fêmea, SEMC. Localidade-tipo: Brasil, Mato Grosso, Chapada (dos Guimarães), e Rio de Janeiro. Distr. Brasil.

walkeri Pont, 1972:54 (nom.n. para maculipennis Walker) (Cyrtoneurina). Distr. Brasil.

\section{Gênero Neomuscina Townsend}

Neomuscina Townsend, 1919:541. Espécie-tipo, cavicola Townsend (des. orig.) = tripunctata Wulp.

atincta Snyder, 1949c:27. Holótipo macho, USNM, parátipos USNM. Localidade-tipo: Panamá, Barro Colorado Is. Distr. Brasil, Costa Rica, Panamá.

atincticosta Snyder, 1949c:37. Holótipo macho, AMNH, parátipos AMNH. Localidade-tipo: Brasil, Santa Catarina, Nova Teutônia. Distr. Brasil.

capalta Snyder, 1949c:17. Holótipo macho, AMNH, parátipo AMNH. Localidade-tipo: Trinidad, Porto Espanha. Distr. Brasil, Ilhas Barro Colorado, Trindade.

currani Snyder, 1949c:35. Holótipo macho, AMNH, parátipos AMNH. Localidade-tipo: Panamá, Barro Colorado Is. Distr. Brasil, Ilhas Barro Colorado.

inflexa Stein, 1918:224 (Cyrtoneurina). Holótipo macho, MSNM. Localidade-tipo: Brasil, São Paulo. Distr. Brazil.

instabilis Snyder, 1949c:30. Holótipo macho, AMNH, Parátipos AMNH/BMNH. Localidade-tipo: Panamá, David. Distr. Brasil, Panamá, Trindade.

mediana Snyder, 1949c:19. Holótipo fêmea, AMNH. Localidade-tipo: Brasil, Guanabara, Rio de Janeiro, Jardim Botânico. Distr. Brasil.

neosimilis Snyder, 1949c:33. Holótipo macho, AMNH, Parátipos AMNH. Localidade-tipo: Brasil, Santa Catarina, Nova Teutônia. Distr. Brasil, Paraguai.

pictipennis Bigot, 1878:39 (Curtonevra). Holótipo fêmea, BMNH. Localidade-tipo: “Brasil”. Distr. Brasil, Costa Rica, El Salvador, Guatemala, Guiana, México, Panamá, Paraguai Peru, Venezuela.

similata Snyder, 1949c:33. Holótipo fêmea, AMNH. Localidade-tipo: Trinidad, Porto Espanha. Distr. Brasil, Guiana, Trindade, Venezuela.

stabilis Stein, 1911:86 (Mydaea). Holótipo macho,

SMT. Localidade-tipo: Peru, nascente do Rio Pachitea. Distr. Brasil, Peru.

vitoriae Lopes \& Khouri, 1995: 51-53, figs. 11-15. Holótipo macho, MNRJ, parátipos MNRJ. Localidade-tipo: Brasil, Minas Gerais. Distr. Brasil. zosteris Shannon \& Del Ponte, 1926:579 (Muscina). Síntipos macho, USNM? Localidade-tipo: Argentina, Tucumán. Distr. Argentina, Brasil.

\section{Gênero Pseudoptilolepis Snyder}

Pseudoptilolepis Snyder, 1949a:14. Espécie-tipo, fulvapoda Snyder (orig. des.).

fluminensis Albuquerque, 1954b:253, figs. 18. Holótipo macho, MNRJ. Localidade-tipo: Brasil, Rio de Janeiro, Nova Friburgo. Distr. Brasil.

fulvapoda Snyder, 1949a:16, figs. 6 e 8. Holótipo macho, AMNH, Parátipos AMNH. Localidade-tipo: Brasil, Santa Catarina, Nova Teutônia. Distr. Brasil, Nicarágua, Venezuela.

Subfamíllia Phaoniinae Malloch, 1917

\section{Gênero Dolichophaonia Carvalho}

Dolichophaonia Carvalho, 1993a:19. Espécie-tipo, Phaonia brasiliensis Albuquerque (des. orig.).

gallicola Albuquerque, 1958c:5, figs. 713 (Phaonia). Holótipo macho, MNRJ, parátipo MNRJ. Localidade-tipo: Brasil, Guanabara, Rio de Janeiro, Represa dos Ciganos. Distr. Brasil.

machadoi Albuquerque, 1958c:10, figs. 1418 (Phaonia). Holótipo macho, MNRJ. Localidade-tipo: Brasil, Guanabara, Rio de Janeiro, Corcovado. Distr. Brasil.

noctiluca Albuquerque, 1958c:28, figs. 3842 (Phaonia). Holótipo macho, MNRJ. Localidade-tipo: Brasil, Rio de Janeiro, Itatiaia. Distr. Brasil.

simplex Albuquerque, 1958c:18, figs. 2427 (Phaonia). Holótipo fêmea, MNRJ. Localidade-tipo: Brasil, Guanabara, Rio de Janeiro, Jardim Botânico. Distr. Brasil.

tachnoides Albuquerque, 1958c:2, figs. 16 (Phaonia). Holótipo macho, MNRJ, parátipo MNRJ. Localidade-tipo: Brasil, Guanabara, Rio de Janeiro. Distr. Brasil.

\section{Gênero Helina Robineau Desvoidy}

Helina Robineau Desvoidy, 1830:493. Espécie-tipo, euphemioidea Robineau Desvoidy (COQUILLETT, 1901:137) = pertusa Meigen.

albuquerquei Pont, 1972:19 (nom.n. para discreta Albuquerque). Distr. Brasil.

discreta Albuquerque, 1956g: 11, figs. 4, 31-37 [Homônio junior secundário de discreta Wulp, 1896]. Holótipo macho, MNRJ, Parátios MNRJ. Localidade-tipo: Brasil, Rio de Janeiro, Teresópolis, Parque Nacional da serra dos Órgãos.

angustipennis Stein, 1911:79 (Mydaea). Síntipos macho, SMT. Localidade-tipo: Peru, Rios Urubamba e Pachitea. Distr. Bolivia, Brasil, Panamá, Peru. 
gigantea Albuquerque, 1956g:15, figs. 5, 38. Holótipo fêmea, MNRJ. Localidade-tipo: Brasil, Rio de Janeiro, Angra dos Reis. Distr Brasil.

nivaloides Albuquerque, 1956g:5, figs. 2, 17-22. Holótipo macho, MNRJ. Localidade-tipo: Brasil, Rio de Janeiro, Itatiaia, Macieira. Distr. Brasil.

nobilis Albuquerque, 1956b:171, figs. 10 18. Holótipo macho, MNRJ, Parátipos MNRJ. Localidade-tipo: Brasil, Guanabara, Rio de Janeiro. Distr. Brasil.

regobarrosi Albuquerque, 1958b:47, figs. 16. Holótipo macho, MNRJ, Parátipos MNRJ. Localidade-tipo: Brasil, Rio de Janeiro, Itatiaia, Lago Azul. Distr. Brasil.

\section{Gênero Phaonia Robineau Desvoidy}

Phaonia Robineau Desvoidy, 1830:482. Espécie-tipo, = viarum Robineau Desvoidy (COQUILLETT, 1901:140) = valida Harris.

annulata Albuquerque, 1957a:1, figs. 1-7 (Bigotomyia). Holótipo macho, MNRJ, Parátipos MNRJ. Localidade-tipo: Brasil, Rio de Janeiro, Itatiaia, Lago Azul. Distr. Brasil

aurantica Albuquerque \& Medeiros, 1980:863, figs. 712 (Bigotomyia). Holótipo macho, MNRJ. Localidade-tipo: Rio de Janeiro, Petrópolis, Alto da Mosela. Distr. Brasil.

bigoti Albuquerque, 1957a:5, figs. 8-12 (Bigotomyia). Holótipo macho, MNRJ. Localidade-tipo: Brasil, Rio de Janeiro, Teresópolis. Distr. Brasil.

grajauensis Albuquerque, 1957a:9, figs. 13-19 (Bigotomyia). Holótipo macho, MNRJ. Localidade-tipo: Brasil, Guanabara, Rio de Janeiro, Grajaú. Distr. Brasil.

grandis Couri, 1982b:45, figs. 1315 (Cyrtoneurina). Holótipo fêmea, MNRJ. Localidade-tipo: Brasil, Rio de Janeiro, Petrópolis, Alto da Mosela. Distr. Brasil.

similata Albuquerque, 1957a:13, figs. 20-25 (Bigotomyia). Holótipo macho, MNRJ. Localidade-tipo: Brasil, Rio de Janeiro, Jussaral, Angra dos Reis. Distr. Brasil.

\section{Gênero Souzalopesmyia Albuquerque}

Souzalopesmyia Albuquerque, 1951a:53. Espécietipo, carioca Albuquerque (orig. des.).

carioca Albuquerque, 1951a:53, figs. 110 e 1315. Holótipo macho, MNRJ, Parátipos MNRJ. Localidade-tipo: Brasil, Guanabara, Rio de Janeiro, Grajaú. Distr. Brasil.

Subfamilia Mydaeinae Verrall, 1888

\section{Gênero Brontaea Kowarz}

Brontaea Kowarz, 1873:461. Espécie-tipo: Anthomyia polystigma Meigen (des. COQUILLETT, 1910:516). debilis Willistaon, 1896:369 (Limnophora). Síntipos macho, BMNH. Localidade-tipo: Ilha Sao Vincente. Distr. Argentina, Brasil, México, Haiti, Jamaica, Porto Rico, Região Neártica, Sao Vincente, Venezuela.

normata Bigot, 1885:272 (Limnophora). Lectótipo macho, UMO, paralectótipo macho, UMO. Localidade-tipo: México. Distr. Bolivia, Brasil, Colombia, México, Paraguai, Peru, Venezuela.

quadristigma Thomson, 1869:551 (Anthomyia). Síntipos macho, NHRS. Localidade-tipo: Ecuador, Puna. Distr. América do Norte, Bolivia, Brasil, Chile, Cuba, Equador, Havai, México, Peru, Porto Rico.

\section{Gênero Graphomya Robineau Desvoidy}

Graphomya Robineau Desvoidy, 1830:403. Espécietipo, Musca maculata Scopoli (WESTWOOD, 1840:141).

analis Macquart, 1851a:228, pl. 23, fig. 9; 1851b:255, pl. 23, fig. 9 (Cyrtonevra). Lectótipo fêmea, MNHN [des. ALBUQUERQUE, 1951b:16]. Localidade-tipo: "Chile” (não Tasmânia como em MACQUART, 1851). Distr. Argentina, Brasil, Chile, Peru.

meridionalis Townsend, 1892:32 (nom.n. for americana Schiner). Distr. Brasil.

americana Schiner, 1868: 304. [homônimo primário júnior de americana Robineau-Desvoidy, 1830. Síntipos macho, NMW. Localidade-tipo: América do Sul”

\section{Gênero Hemichlora Wulp}

Hemichlora Wulp, 1896a:303. Espécie-tipo, Curtonevra vittigera Bigot (mon.) = scordalus Walker.

scordalus Walker, 1861:313 (Pyrellia). Holótipo fêmea, BMNH. Localidade-tipo: "México”. Distr. Brasil Costa Rica, Guatemala, México.

\section{Gênero Mydaea Robineau Desvoidy}

Mydaea Robineau Desvoidy, 1830:479. Espécie-tipo, scutellaris Robineau Desvoidy (COQUILLETT, 1901:139) = corni Scopoli.

castanea Albuquerque, 1955c:377, figs. 1420 . Holótipo macho, MNRJ. Localidade-tipo: Brasil, Guanabara, Rio de Janeiro, Tijuca. Distr. Brasil.

plaumanni Snyder, 1941:3. Holótipo fêmea, AMNH, Parátipos USNM/AMNH. Localidade-tipo: Brasil, Santa Catarina, Nova Teutônia. Distr. Brasil, México, Paraguai.

\section{Gênero Myospila Rondani}

Myospila Rondani, 1856:91. Espécie-tipo, Musca meditabunda Fabricius (des. orig.).

fluminensis Couri \& Lopes, 1988:4, figs. 8 10. Holótipo fêmea, MNRJ, Parátipos MNRJ. Localidade-tipo: Brasil, Rio de Janeiro, Petrópolis. Distr. Brasil. 
obscura Shannon \& Del Ponte, 1926:580 (Muscina). Holótipo fêmea, USNM. Localidade-tipo: Argentina, Buenos Aires. Distr. Argentina, Brasil, Peru.

pallidicornis Bigot, 1887:clxxiv (também 1888:614) (Curtonevra). Lectótipo fêmea, BMNH. Localidade-tipo: “Mexique”. Distr. Bolivia, Brasil, Costa Rica, Cuba, Guiana, Haiti, Jamaica, México, Panamá, Peru, Porto Rico, Sao Domingo, , Venezuela.

Subfamília Coenosiinae Verrall, 1888

Tribo Limnophorini Villeneuve, 1902

Gênero Agenamyia Albuquerque

Agenamyia Albuquerque, 1953:519. Espécie-tipo, fumipennis Albuquerque (des. orig.).

fumipennis Albuquerque, 1953:519, figs. 112. Holótipo macho, MNRJ. Localidade-tipo: Brasil, Guanabara, Rio de Janeiro, Gávea. Distr. Brasil.

\section{Gênero Limnophora Robineau Desvoidy}

Limnophora Robineau Desvoidy, 1830:517. Espécietipo, palustris Robineau Desvoidy (COQUILLETT, 1910:561) = maculosa (Meigen).

altaneira Albuquerque, 1954d:400, figs. 1015 (Heliographa). Holótipo macho, MNRJ. Localidade-tipo: Brasil, Minas Gerais, Serra do Cipó. Distr. Belize, Brasil, Venezuela.

longiseta Lopes \& Khouri, 1989:335, figs. 27 (Heliographa). Holótipo macho, MNRJ, Parátipos MNRJ. Localidade-tipo: Brasil, Rio de Janeiro, Petrópolis, Alto da Mosela. Distr. Brasil.

nigrargentata Albuquerque, 1954d:404, figs. 1622 (Heliographa). Holótipo macho, MNRJ. Localidade-tipo: Brasil, Rio de Janeiro, Teresópolis. Distr. Brasil.

piliseta Stein, 1919:136 (nom.n. for plumiseta Stein, 1911). Distr. Argentina, Brasil, Guiana, Peru.

plumiseta Stein, 1911: 177 [homônimo primário júnior de plumiseta Stein, 1903]. Síntipo macho, SMT. Localidadetipo: Peru (foz do Rio Pachitea)

saeva Wiedemann, 1830:430 (Anthomyia). Lectótipo macho, NMW [des. PONT, 1997]. Localidade-tipo: "Brasil”. Distr. Argentina, Bolivia, Brasil, Cost Rica, Equador, Guatemala, Guiana, México, Panamá, Peru, Venezuela.

spreta Malloch, 1921:422. Holótipo macho, BMNH, Parátipos BMNH. Localidade-tipo: Brasil, Teresópolis. Distr. Brasil.

vittata Macquart, 1851a:230, pl. 23, fig. 11; 1851b:257, pl. 23, fig.11 (Microchylum). Lectótipo macho, MNHN [des. ALBUQUERQUE, 1950:2], Paralectótipo MNHN. Localidadetipo: Brasil, Bahia. Distr. Argentina, Brasil.
Gênero Lispe Latreille

Lispe Latreille, 1797:169. Espécie-tipo, Musca tentaculata De Geer (sub. mon., LATREILLE, 1802:462).

latana Snyder, 1949b:4. Holótipo macho, AMNH, Parátipos BMNH. Localidade-tipo: Brasil, Natal. Distr. Brasil.

serotina Wulp, 1896b:342. Lectótipo macho, BMNH, Paralectótipo BMNH. Localidade-tipo: México, Veracruz, Atoyac. Distr. Brasil, Guiana, México, Paraguai, Porto Rico.

\section{Gênero Lispoides Malloch}

Lispoides Malloch, 1920:146. Espécie-tipo, Limnophora aequifrons Stein (des. orig.).

lopesi Albuquerque, 1955a:153, figs. 1 9. Holótipo macho, MNRJ, Parátipos MNRJ. Localidade-tipo: Brasil, Rio de Janeiro, Teresópolis. Distr. Brasil.

\section{Gênero Pachyceramyia Albuquerque}

Pachyceramyia Albuquerque, 1955b:253. Espécietipo, enigmatica Albuquerque (orig. des.).

enigmatica Albuquerque, 1955b:253, figs. 16 . Holótipo fêmea, MNRJ. Localidade-tipo: Brasil, Guanabara, Rio de Janeiro, Manguinhos. Distr. Brasil.

\section{Gênero Spilogona Schnabl}

Limnophora, subg. Spilogona Schnabl, 1911:92. Espécie-tipo, Aricia carbonella Zetterstedt (mon.).

golbachi Snyder, 1957:469. Holótipo macho, IMLA, Parátipos IMLA/AMNH. Localidade-tipo: Argentina, Tucumán, Queb. La Toma. Distr. Argentina, Brasil, Colombia.

\section{Tribe Coenosiini}

\section{Gênero Bithoracochaeta Stein}

Bithoracochaeta Stein, 1911:177. Espécie-tipo, Anthomyia despecta Walker (orig. des.) = leucoprocta Wiedemann.

annulata Stein, 1911:178. Síntipos macho/fêmea, SMT. Localidade-tipo: Peru, Valley de Lares e foz do Rio Pachitea. Distr. Brasil, Peru, Suriname.

calopus Bigot, 1885:275 (?Hydrophoria). Holótipo fêmea, UMO. Localidade-tipo: “Mexico”. Distr.- Argentina, Brasil, Mexico.

leucoprocta Wiedemann, 1830:433 (Anthomyia). Lectótipo fêmea, UZMC [des. PONT, 1997]. Localidade-tipo: West Indies. Distr. América do Norte, Argentina, Brasil, Cuba, Guiana, Jamaica, México, Paraguai, Peru, Porto Rico, Uruguai, Venezuela. 
maricaensis Couri \& Motta, 1995: 211, figs 1-9. Holótipo macho, MNRJ. Localidade-tipo: Brasil, Rio de Janeiro. Distr. Brasil.

\section{Gênero Coenosia Meigen}

Coenosia Meigen, 1826:210. Espécie-tipo, Musca tigrina Fabricius (WESTWOOD, 1840:143).

camorinensis Albuquerque, 1956d:8, figs. 1718. Holótipo fêmea, MNRJ, Parátipos MNRJ. Localidade-tipo: Brasil, Guanabara, Rio de Janeiro, Jacarepaguá, Represa do Camorim. Distr. Brasil.

curviventris Albuquerque, 1959:1, figs. 18 . Holótipo macho, MNRJ, Parátipos MNRJ. Localidade-tipo: Brasil, Rio de Janeiro, Teresópolis, Parque Nacional da Serra dos Órgãos. Distr. Brasil.

enormis Albuquerque, 1956b:5, figs. 10-12. Holótipo fêmea, MNRJ, Parátipos MNRJ. Localidade-tipo: Brasil, Rio de Janeiro, Itatiaia. Distr. Brasil.

latitibia Albuquerque, 1957b:362, figs. 10 17. Holótipo macho, MNRJ, Parátipos MNRJ. Localidade-tipo: Brasil, Guanabara, Rio de Janeiro. Distr. Brasil.

leucotrichia Albuquerque, 1956b:1, figs. 1-5. Holótipo macho, MNRJ. Localidade-tipo: Brasil, Rio de Janeiro, Itatiaia, Maromba. Distr. Brasil.

longipede Albuquerque, 1956b:10, figs. 20-27. Holótipo macho, MNRJ, Parátipos MNRJ. Localidade-tipo: Brasil, Rio de Janeiro, Teresópolis. Distr. Brasil.

minuscularis Albuquerque, 1956d:5, figs. 916. Holótipo macho, MNRJ. Localidade-tipo: Brasil, Guanabara, Rio de Janeiro, Grajaú. Distr. Brasil.

tinctipenis Albuquerque, 1956f:3, figs. 6-9. Holótipo fêmea, MNRJ. Localidade-tipo: Brasil, Rio de Janeiro, Teresópolis, Parque Nacional da Serra dos Órgãos. Distr. Brasil.

trichophthalma Albuquerque, 1959:5, figs. 914. Holótipo macho, MNRJ, Parátipos MNRJ. Localidade-tipo: Brasil, Rio de Janeiro, Teresópolis, Parque Nacional da Serra dos Órgãos. Distr. Brasil.

\section{Gênero Cordiluroides Albuquerque}

Cordiluroides Albuquerque, 1954c:361. Espécie-tipo, listarata Albuquerque (orig. des.).

listrata Albuquerque, 1954c:364, figs. 8 15. Holótipo macho, MNRJ, Parátipos MNRJ. Localidade-tipo: Brasil, Rio de Janeiro. Distr. Brasil.

megalopyga Albuquerque, 1954c:362, figs. 17. Holótipo macho, MNRJ, Parátipos MNRJ. Localidade-tipo: Brasil, Rio de Janeiro, Novo Friburgo. Distr. Brasil.

Gênero Insulamyia Couri
Insulamyia Couri, 1982a:29. Espécie-tipo, inusitata Couri (mon.).

inusitata Couri, 1982a:29, figs. 14 . Holótipo fêmea, MNRJ. Localidade-tipo: Rio de Janeiro, Ilha do Governador. Distr. Brasil.

\section{Gênero Neodexiopsis Malloch}

Neodexiopsis Malloch, 1920:162. Espécie-tipo, Dexiopsis basalis Stein (orig. des.).

alacris Couri \& Albuquerque, 1979:500, fig. 1. Holótipo fêmea, MNRJ, Parátipos MNRJ. Localidade-tipo: Brasil, Rio de Janeiro, Petrópolis, Le Vallon, Alto da Mosela. Distr. Brasil.

albisquama Albuquerque, 1959:9, figs. 1519 (Austrocoenosia). Holótipo macho, MNRJ, Parátipos MNRJ. Localidade-tipo: Brasil, Rio de Janeiro, Teresópolis, Parque Nacional da Serra dos Órgãos. Distr. Brasil.

barbiventris Couri \& Albuquerque, 1979:502, figs. 2 6. Holótipo macho, MNRJ, Parátipos MNRJ. Localidadetipo: Rio de Janeiro, Itatiaia, Lago Azul. Distr. Brasil.

calopyga Loew, 1872:94 (Coenosia). Síntipos macho, MCZ/ZMHU. Localidade-tipo: EUA, Pennsylvania. Distr. América do Norte, Brasil, Peru.

cirratipila Snyder, 1957:463. Holótipo macho, IMLA, Parátipos IMLA. Localidade-tipo: Argentina, Tucumán, Burruyacu, V. Padre Monti. Distr. Argentina, Brasil.

elegans Couri \& Albuquerque, 1979:503, fig. 7. Holótipo fêmea, MNRJ, paratype MNRJ. Localidade-tipo: Rio de Janeiro, Petrópolis, Alto da Mosela. Distr. Brasil.

flavipalpis Albuquerque, 1956c:198, figs. 813. Holótipo macho, MNRJ. Localidade-tipo: Brasil, Rio de Janeiro, Itatiaia, Lago Azul. Distr. Brasil.

fulvifrontis Couri \& Albuquerque, 1979:505, fig. 9. Holótipo fêmea, MNRJ, Parátipos MNRJ. Localidade-tipo: Rio de Janeiro, Petrópolis, Le Vallon, Alto da Mosela. Distr. Brasil.

itatiaiensis Albuquerque, 1954a:184, figs. 2730 (Austrocoenosia). Holótipo fêmea, MNRJ, Parátipos MNRJ. Localidade-tipo: Brasil, Rio de Janeiro, Itatiaia, Macieira. Distr. Brasil.

latimaculata Albuquerque, 1956a:74, figs. 59 (Austrocoenosia). Holótipo macho, MNRJ. Localidade-tipo: Brasil, Rio de Janeiro, Juçaral, Angra dos Reis. Distr. Brasil.

mesofulvata Albuquerque, 1959:13, figs. 2026 (Austrocoenosia). Holótipo macho, MNRJ. Localidade-tipo: Brasil, Rio de Janeiro, Teresópolis, Parque Nacional da Serra dos Órgãos. Distr. Brasil.

parvula Albuquerque, 1958a:269, figs. 712 . Holótipo macho, MNRJ, Parátipos MNRJ/BMNH. Localidade-tipo: Brasil Rio de Janeiro, Petrópolis, Alto da Mosela, Le Vallon. Distr. Brasil. 
pectinata Couri \& Albuquerque, 1979:510. Holótipo fêmea, MNRJ, Parátipos MNRJ. Localidade-tipo: Rio de Janeiro, Petrópolis, Alto da Mosela, Le Vallon. Distr. Brasil. rufipes Macquart, 1851a:245, pl. 25, fig. 3; 1851b:272, pl. 25, fig. 3 (Choetura). Lectótipo macho, MNHN [des. ALBUQUERQUE, 1949:440]. Localidade-tipo: Uruguai, Maldonado. Distr. Brasil, Uruguai.

rustica Albuquerque, 1956f:7, figs. 13-19 (Austrocoenosia). Holótipo macho, MNRJ. Localidade-tipo: Brasil, Rio de Janeiro, Itatiaia, Lago Azul. Distr. Brasil.

setipuncta Snyder, 1957:455. Holótipo macho, IMLA, Parátipos USNM/IMLA. Localidade-tipo: Argentina, Tucumán, Queb. La Toma. Distr. Argentina.

sima Snyder, 1957:462. Holótipo macho, IMLA, Parátipos IMLA. Localidade-tipo: Argentina, Tucumán, San Javier. Distr. Argentina.

subtilis Couri \& Albuquerque, 1979:512, fig. 20. Holótipo fêmea, MNRJ, Parátipos MNRJ. Localidade-tipo: Brasil, Rio de Janeiro, Itatiaia, Lago Azul. Distr. Brasil.

tinctifacies Albuquerque, 1958a:265, figs. 16 (Austrocoenosia). Holótipo macho, MNRJ. Localidade-tipo: Brasil, Rio de Janeiro, Petrópolis, Alto da Mosela, Le Vallon. Distr. Brasil.

vulgaris Couri \& Albuquerque, 1979:513, fig.22. Holótipo fêmea, MNRJ, Parátipos MNRJ. Localidade-tipo: Brasil, Rio de Janeiro, Petrópolis, Alto da Mosela. Distr. Brasil.

xanthopoda Albuquerque, 1956d:1, figs. 18 (Austrocoenosia). Holótipo macho, MNRJ, Parátipos MNRJ. Localidade-tipo: Brasil, Guanabara, Rio de Janeiro, Represa do Camorim. Distr. Brasil.

\section{Gênero Pilispina Albuquerque}

Pilispina Albuquerque, 1954a:180. Espécie-tipo, pilitibia Albuquerque (orig. des.)

pilitibia Albuquerque, 1954a:180, figs. 10 18. Holótipo macho, MNRJ. Localidade-tipo: Brasil, Rio de Janeiro, Itatiaia. Distr. Brasil.

\section{Gênero Plumispina Albuquerque}

Plumispina Albuquerque, 1954a:177. Espécie-tipo, longipilis Albuquerque (orig. des.).

longipilis Albuquerque, 1954a:177, figs. 19 (Plumispina). Localidade-tipo: Brasil, Guanabara, Rio de Janeiro, Grajaú. Distr. Brasil.

\section{Agradecimentos}

MSC e CJBC agradecem o apoio financeiro do Conselho Nacional de Desenvolvimento Científico e Tecnológico, CNPq (processos 300386-80 e 304148/ 2002-4 respectivamente).

\section{Referências Bibliográficas}

ALBUQUERQUE, D. de O. 1950. Primeiras notas sobre alguns Muscidae de Macquart, da coleção do Museu de Paris. Bol. Mus. nac. Rio de J. (Zoologia) 98:1-7.

ALBUQUERQUE, D. de O. 1951a. Sobre um gênero e duas espécies novas de "Phaoniinae” neotropical (Diptera, Muscidae). Rev. bras. Biol. 11:53 57.

ALBUQUERQUE, D. de O. 1951b. Quinta nota sôbre tipos de Macquart (Diptera Muscidae), existentes no Museu Nacional de História Natural de Paris e Descrição de uma espécie nova, proveniente do Chile. Bol. Mus. nac. Rio de J. (Zoologia) 105:1-17.

ALBUQUERQUE, D. de O. 1952. Sôbre alguns gêneros metálicos de Phaoninae Neotropicais e descrição de uma Xenomorellia nova (Diptera: Muscidae). Bol. Mus. nac. Rio de J. (Zoologia) 116:1-21.

ALBUQUERQUE, D. de O. 1953. Fauna do Distrito Federal. VII. Sôbre um Novo Gênero e Espécie Nova de Limnophorini, com Descrição do Alótipo Fêmea de “Coenosopsia prima” Mall., capturada no Rio de Janeiro (Diptera Muscidae). Anais Acad. bras. Cienc. 25:519 525.

ALBUQUERQUE, D. de O. 1954a. Fauna do Distrito Federal. XV: Descrição de dois gêneros e quatro espécies novas de Coenosiinae (Diptera, Muscidae). Rev. bras. Biol. 14:177186.

ALBUQUERQUE, D. de O. 1954b.Contribuição ao conhecimento do gênero Pseudoptilolepis Snyder, 1949 (Diptera, Muscidae). Rev. bras. Biol. 14:253 256.

ALBUQUERQUE, D. de O. 1954c. Fauna do Distrito Federal. XXI: Um gênero e três espécies novas de Coenosiinae (Diptera, Muscidae). Rev. bras. Biol. 14: 361369.

ALBUQUERQUE, D. de O. 1954d. Fauna do Distrito Federal. XVIII Sôbre três espécies de Heliographa Malloch, 1921 (Diptera Muscidae). Anais Acad. bras. Cienc. 26:395 409.

ALBUQUERQUE, D. de O. 1955a. Uma nova espécie de “Lispoides” Malloch, 1920 (Diptera, Muscidae). Rev. bras. Biol. 15:153156.

ALBUQUERQUE, D. de O. 1955b - Fauna do Distrito Federal. XXXII. Um gênero e uma espécie nova de Phaoninae (Diptera, Muscidae). Rev. bras. Biol. 15:253 256.

ALBUQUERQUE, D. de O. 1955c. Fauna do Distrito Federal: XXXV. Três novas espécies de Phaoninae (Diptera Muscidae). Anais Acad. bras. Cienc. 27:373 380.

ALBUQUERQUE, D. de O. 1956a.Redescrição de Caricea plumiseta (Stein, 1911) e descrição de duas espécies novas de Austrocoenosia Mall., 1934 (Diptera-Muscidae). Rev. bras. Ent. 5:71 80.

ALBUQUERQUE, D. de O. 1956b. Fauna do Distrito Federal: XXXIX. Duas novas espécies de Helina R. D. (Diptera Muscidae). Rev. bras. Ent. 5:167 176. 
ALBUQUERQUE, D. de O. 1956c. Duas espécies novas de Neodexiopsis Mall., 1920 (Diptera, Muscidae). Rev. bras. Biol. 16:195200.

ALBUQUERQUE, D. de O. 1956d. Fauna do Distrito Federal. XXX. Três espécies novas de Coenosiinae (Diptera, Muscidae). Bol. Mus. nac. Rio de J. (Zoologia) 133:1-11.

ALBUQUERQUE, D. de O. 1956e. Fauna do Distrito Federal. XIII. Sôbre o gênero Polietina Schnable [sic] \& Dziedzicki, 1911, com descrições de espécies novas (Diptera Muscidae). Bol. Mus. nac. Rio de J. (Zoologia) 139:1-31.

ALBUQUERQUE, D. de O. 1956f. Novas espécies de Coenosiinae (Diptera Muscidae). Bol. Mus. nac. Rio de J. (Zoologia) 146:1-23.

ALBUQUERQUE, D. de O. 1956g. Fauna do Distrito Federal. XLI. Redescrição de Helina angustipennis (Stein, 1911) e descrição de quatro espécies novas (Diptera Muscidae). Bol. Mus. nac. Rio de J. (Zoologia) 148:1-33.

ALBUQUERQUE, D. de O. 1957a. Fauna do Distrito Federal. XXXVI. Novas espécies de Bigotomyia Malloch, 1921 (Diptera Muscidae). Bol. Mus. nac. Rio de J. (Zoologia) 159:1-17.

ALBUQUERQUE, D. de O. 1957b. Um gênero e duas novas espécies de Coenosiinae do Brasil (Diptera, Muscidae). Rev. bras. Biol. 17:359 365.

ALBUQUERQUE, D. de O. 1957c. Fauna do Distrito Federal. XLI. Notas sôbre alguns Muscídeos, com descrição de dois alótipos (Diptera Muscidae). Bol. Mus. nac. Rio de J. (Zoologia) 164:1-13.

ALBUQUERQUE, D. de O. 1958a. Duas Espécies Novas de Coenosiinae (Dipt. Muscidae). Stud. entomol., Brasília 1:265272.

ALBUQUERQUE, D. de O. 1958b. Sôbre uma nova espécie de Helina R. D., 1830 e notas sôbre Helina pleuraseta Snyder, 1940 (Diptera, Muscidae). Rev. bras. Ent. 8:47 54.

ALBUQUERQUE, D. de O. 1958c. Fauna do Distrito Federal. V. Contribuição ao conhecimento de Phaonia R. D., 1830, com descrição de novas espécies (Diptera Muscidae). Bol. Mus. nac. Rio de J. (Zoologia) 179:1-38.

ALBUQUERQUE, D. de O. 1958d. Sôbre Ophyra R. D., 1830 na América do Sul, com descrição de uma espécie nova (Diptera Muscidae). Bol. Mus. nac. Rio de J. (Zoologia) 181:1-13.

ALBUQUERQUE, D. de O. 1959. Novas espécies de Coenosiinae (Diptera Muscidae). Bol. Mus. nac. Rio de J. (Zoologia) 193:1-17.

ALBUQUERQUE, D. de O. \& MEDEIROS, K. F., 1980. Estudos sobre Bigotomyia Malloch, 1921, com descrição de 4 espécies novas (Diptera, Muscidae). Rev. bras. Biol. 40:861-879.
BIGOT, J. M. F. 1878. Diptères nouveaux ou peu connus. 9e partie. XII. Gênero Phumosia, Pyrellia, Cosmina, Ochromyia et Curtonevra. XIII. Genres Ocyptera (Latr.), Ocypterula, Exogaster (Rond.). XIV. Notes et mélanges. Ann. Soc. ent. Fr. 8:31 48.

BIGOT, J. M. F. 1885. Diptères nouveaux ou peu connus. 25e partie. XXXIII. Anthomyzides nouvelles. Ann. Soc. ent. Fr. 4:263 304.

BIGOT, J. M. F. 1887. [Note, in Séance de 9 November 1887.] Bull. Soc. ent. Fr. 21:1522-1524.

BIGOT, J. M. F. 1888. Diptères nouveaux ou peu connus. Muscidi (J.B.). Bull. Soc. zool. Fr. 12:581 617.

BRAUER, F. \& BERGENSTAMM, J. von 1889. Die Zweiflügler des Kaiserlichen Museums zu Wien. IV. Vorarbeiten zu einer Monographie der Muscaria Schizometopa (exclusive Anthomyidae). Pars I. Denks.Akad. Wissensch. 56:69 180.

BRAUER, F. \& BERGENSTAMM, J. von 1893. Die Zweiflügler des Kaiserlichen Museums zu Wien. VI. Vorarbeiten zu einer Monographie der Muscaria Schizometopa (exclusive Anthomyidae). Pars III. Denks.Akad. Wissensch. 60: 89240.

CARVALHO, C.J.B. de 1989a. Classificação de Muscidae (Diptera): uma proposta através da análise cladística. Rev. bras. Zool. 6:627 648.

CARVALHO, C.J.B. de 1993a. Dolichophaonia, gen.n. (Diptera, Muscidae, Phaoniinae): descrições, novas combinações, sinonímias e chave para as espécies. Rev. bras. Ent. 37:19-34.

-- CARVALHO, C.J.B. de 1993b. Micropotamia, gen.n. of Neotropical Muscidae (Diptera, Azeliinae), with comments on allied Azeliini genera. Rev. bras. Zool. 9:241-246.

CARVALHO, C.J.B. de \& COURI, M.S. 2002. Part I. Basal groups. In Carvalho, C.J.B. de (Ed) Muscidae (Diptera) of the Neotropical Region: taxonomy. Editora Universidade Federal do Paraná, Curitiba, p.17-132.

CARVALHO, C.J.B. de, COURI M. S.; PONT A. C.; PAMPLONAD. \& LOPES S. M. 1993. Part II. Muscidae. In Carvalho, C.J.B. de (ed.). A catalogue de the Fanniidae e Muscidae de the Neotropical region. Sociedade Brasileira de Entomologia, Curitiba, p.1-201.

CARVALHO, C.J.B. de, COURI M. S.; PONT A. C.; PAMPLONAD. \& LOPES S. M. 2005. A Catalogue of the Muscidae (Diptera) of the Neotropical Region. Zootaxa 860:1-282.

COQUILLETT, D.W. 1901. Types de Anthomyid Genera. J. N. Y. ent. Soc. 9:134146.

COQUILLETT, D.W. 1910. The type-species of the North American Genera of Diptera. Proc.U. S. nat. Mus. 37:499647 
COURI, M.S. 1982a. Insulamyia, gen.n., e notas sobre Spathipheromyia Bigot, 1884 (Diptera, Muscidae, Coenosiinae). Rev. bras. Ent. 26:29-36.

COURI, M.S. 1982b. Adenda ao trabalho de Snyder (1954) sobre Cyrtoneurina Giglio-Tos, 1893 (Diptera, Muscidae, Cyrtoneurininae). Rev. bras. Ent. 26:37-53.

COURI, M.S. 1983. Descrição de 6 espécies novas de Philornis Meinert, 1890 (Diptera, Muscidae, Cyrtoneurininae). Rev. bras. Biol. 43:297-309.

COURI, M.S. 1984. Notes and descriptions of Philornis flies (Diptera, Muscidae, Cyrtoneurininae). Revta bras. Ent. 28:473-490.

COURI, M. S. \& ALBUQUERQUE, D. de O. 1979. Estudos sobre Neodexiopsis Malloch, 1920 com notas nomenclaturais sobre Coenosiinae (Diptera, Muscidae). Rev. bras. Biol. 39:499-517.

COURI, M.S. \& CARVALHO, C.J.B. de 1996. Duas novas espécies de Polietina Schnabl \& Dziedzicki (Diptera, Muscidae) do Brasil. Rev. bras. Zool. 12:825-831.

COURI, M. S. \& CARVALHO, C. J. B. de 2002.. Part II. Apical Groups. In Carvalho, C.J.B. de (Ed) Muscidae (Diptera) of the Neotropical Region: taxonomy. Editora Universidade Federal do Paraná, Curitiba, p.133-262.

COURI, M.S. \& LOPES, S.M. 1988. Duas espécies novas de Myospila Rondani, 1856 do Brasil (Diptera, Muscidae, Mydaeinae). Bol. Mus. nac. Rio de J. (Zoologia) 321:1-11.

COURI, M.S. \& MACHADO, A. 1990. Descrição de Cyrtoneurina prima, sp. nov. (Diptera, Muscidae, Reinwardtiinae). Rev. bras. Ent. 34:659-662.

COURI, M. S. \& MOTTA, H. C. G. 1995. Uma nova espécie de Bithoracochaeta Stein,. 1911 (Diptera, Muscidae, Coenosiinae). Rev. bras. Zool. 12:123-125.

CURRAN, C.H. 1939. Two new American Diptera with notes on Asemosyrphus Bigot. Amer. Mus. Novit. 1031:1-3.

CURTIS, J. 1839. British Entomology; being illustrations and descriptions of the genera of insects found in Great Britain and Irele: containing Coloured Figures from Nature of the most rare and beautiful species, and in many instances of the plants upon which they are found. Volume 16, unnumbered.

DODGE, H.R. 1963. A new Philornis with coprophagous larva, and some related species (Diptera: Muscidae). J. Kans. ent. Soc. 36:239 247.

DODGE, H.R. 1968. Some new and little known species of Philornis (Diptera: Muscidae). J. Kans. Ent. Soc. 41: 155164.

DODGE, H.R. \& AITKEN, T.H.G. 1968. Philornis flies from Trinidad(Diptera, Muscidae). J. Kans. Ent. Soc. 41:134154.

FALLÉN, C.F. 1817. Beskrifning Öfver de i Sverige funna Fluge Arter, som kunna föras till Slägtet Musca. Första Afdelningen. K. svenska VetenskAkad. Handl.:226 254.
GEDEFROY, E.L. 1762. Histoire abrégée des Insectes qui se trouvent aux environs de Paris; dans laquelle ces Animaux sont rangés suivant un ordre méthodique. 2, p.1-690.

GIGLIO TOS, H. 1893. Diagnosi di nuovi generi e di nuove specie di Ditteri. VIII. Boll. Mus. Zool. Anat. Comp. Torino 8:1-11.

GREENBERG, B. 1971. Flies e Disease. Volume 1. Ecology, Classification and Biotic Associations. University Press, Princeton.

GUIMARÃES, J.H. \& PAPAVERO, N. 1999. Myiasis in man and animals in the Neotropical Region; bibliographic database. Plêiade/FAPESP, São Paulo.

HENNIG, W.1956. Muscidae [Part, Lieferung 194]. In Lindner, E. (Ed.). Die Fliegen der palaearktischen Region, 63b, p.97-144.

HENNIG, W.1957a. Muscidae [Part, Lieferung 197]. In Lindner, E. (Ed.). Die Fliegen der palaearktischen Region, 63b, p.145-192.

HENNIG, W.1957b. Los Insectos de las Islas Juan Ferneez. 34. Therevidae, Helomyzidae, Piophilidae, Milichiidae und Muscidae (Diptera). (Nachtrag.) Rev. chil. Ent. 5: 409412.

HENNIG, W.1958. Muscidae [Part, Lieferung 199]. In Lindner, E. (Ed.). Die Fliegen der palaearktischen Region, 63b, p.193-232.

HENNIG, W.1959. Muscidae [Part, Lieferung 204]. In Lindner, E. (Ed.). Die Fliegen der palaearktischen Region, 63b, p.233-288.

HENNIG, W.1960a. Muscidae [Part, Lieferung 209]. In Lindner, E. (Ed.). Die Fliegen der palaearktischen Region, 63b, p.385-432.

HENNIG, W.1960b. Muscidae [Part, Lieferung 213]. In Lindner, E. (Ed.). Die Fliegen der palaearktischen Region, 63b, p.433-480.

HENNIG, W.1962a. Muscidae [Part, Lieferung 225]. In Lindner, E. (Ed.). Die Fliegen der palaearktischen Region, 63b, p.625-672.

HENNIG, W.1962b. Muscidae [Part, Lieferung 223]. In Lindner, E. (Ed.). Die Fliegen der palaearktischen Region, 63b, p. 577-624.

HENNIG, W.1962c. Muscidae [Part, Lieferung 227]. In Lindner, E. (Ed.). Die Fliegen der palaearktischen Region, 63b, p.673-720.

HENNIG, W.1962d. Muscidae [Part, Lieferung 229]. In Lindner, E. (Ed.). Die Fliegen der palaearktischen Region, 63b, p.721-768.

HENNIG, W.1963a. Muscidae [Part, Lieferung 233]. In Lindner, E. (Ed.). Die Fliegen der palaearktischen Region, 63b, p.769-816.

HENNIG, W.1963b. Muscidae [Part, Lieferung 242]. In Lindner, E. (Ed.). Die Fliegen der palaearktischen Region, 63b, p.913-960. 
HENNIG, W.1964a. Muscidae [Part, Lieferung 248]. In Lindner, E. (Ed.). Die Fliegen der palaearktischen Region, 63b, p.961-1008.

HENNIG, W.1964b. Muscidae [Part, Lieferung 249]. In Lindner, E. (Ed.). Die Fliegen der palaearktischen Region, 63b, p.1009-1056.

HENNIG, W.1965. Vorarbeiten zu einem phylogenetischen System der Muscidae (Diptera: Cyclorrhapha). Stuttg. Beitr. Naturkd. 141, p.1-100.

HOUGH, G. de N. 1900. South American Muscidae in the Collection of S. W. Willistaon. Kans. Univ. Quart. 9:203 232.

I.C.Z.N. 1925. Opinion 82. Opinions rendered by the International Commission on Zoological Nomenclature. Opinion 82. Suspension of Rules for Musca Linnaeus, 1758A, Type M. domestica. Smithson. Misc. Collec. 75:1-7.

I.C.Z.N. 1957. Opinion 441. Validation under the Plenary Powers of the names for five genera in the Order Diptera (Class Insecta) published in 1762 by Gedefroy (E. L.) in the work entitled Histoire abrégée des Insectes qui se trouvent aux Environs de Paris. (Opinion supplementary to Opinion 228). Opin. Decl. Rend. Int. Comm. Zool. Nomencl. 15:83120.

I.C.Z.N. 1974. Opinion 1008. Siphona Meigen, 1803 and Haematobia Lepeletier \& Serville, 1828 (Insecta: Diptera): designations of type-species under the plenary powers. Bull. zool. Nom. 30:157-158.

KOWARZ, F. 1873. Beitrag zur Dipteren-Fauna Ungarns. Verhandlungen der zoologisch-botanischen Gesellschaft in 23:453-464.

LATREILLE, P.A. 1797. Précis des caractères génériques des Insectes, disposés dans un ordre naturel. Paris \& Bourdeaux.

LATREILLE, P.A. 1802. Histoire naturelle, générale et particulière, des Crustacés et des Insectes. Volume 3. Paris.

LATREILLE, P.A.;A.L.M. LEPELETIER; J.G.A. SERVILLE \& F.E. GUÉRIN MENEVILLE. 1828. Entomologie, ou histoire naturelle des Crustacés, des Arachnides et des Insectes. In Encyclopédie méthodique, Histoire naturelle, Volume 10, p.1-833.

LINNAEUS, C. 1758. Systema Naturae per regna tria naturae, secundum classes, ordines, genera, species, cum caracteribus, differentiis, sinonymis, locis. 10th Edition. Volume 1. Holmiae [= Stockholm].

LOEW, H. 1872. Diptera Americae septentrionalis indigena. Centuria decima. Berl. ent. Z. 16:49-124.

LOPES, H. de S. 1938. Sur une espèce du genre Charadrella Wulp (Dipt. Anthomyiidae) trouvée au Brésil et vivant aux dépens de Bulinus taunaysi Fer. (Mollusca Pulmonata). Compte rendu séances Soc. Biol. 128:926928.

LOPES, S.M. 1985. Descrição de Ophyra albuquerquei, sp.n. (Diptera, Muscidae, Muscinae). Rev. bras. Biol. 45:117-119.
LOPES, S.M. \& KHOURI, A. 1989. Notas sobre Limnophorinae (Diptera, Muscidae) com descrição de uma espécie nova no Brasil. Mem. Inst. Osw. Cruz 84, Suppl. 4:335-339.

LOPES, S.M. \& Khouri,A. 1995. Espécies novas de Neomuscina Townsend, 1919 (Diptera: Muscidae, Azelinae, Reinwardtiini). Bol. Mus. ent. Univ. Valle 3:45-53.

MACQUART, J. 1846a. Diptères Exotiques nouveaux ou peu connus. Supplément. Mém. Soc. Scienc. Agric. Arts Lille 1844:133364.

MACQUART, J. 1846b. Ibidem, reprinted with pagination 5238.

MACQUART, J. 1851a. Diptères Exotiques nouveaux ou

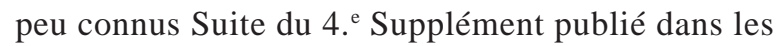
mémoires de 1849 [part]. Mém. Soc. Scienc. Agric. Arts Lille 1850:134 294.

MACQUART, J. 1851b. Ibidem, reprinted with pagination 161336.

MALLOCH, J.R. 1920. Descriptions of New North American Anthomyiidae (Diptera). Trans. Amer. ent. Soc. 46:133196.

MALLOCH, J.R. 1921. Exotic Muscaridae (Diptera).- IV. Ann. Mag. nat. Hist. 8:414 425.

MALLOCH, J.R. 1923. Exotic Muscaridae(Diptera).- XI. Ann. Mag. nat. Hist. 12:505 528.

MALLOCH, J.R. 1925. Exotic Muscaridae (Diptera).- XVI Ann. Mag. nat. Hist. 16:81 100.

MALLOCH, J.R. 1934. Muscidae. In Diptera de Patagonia e South Chile 7:171346.

MEIGEN. J.W. 1826. Systematische Beschreibung der bekannten europäischen zweiflügeligen Insekten. Volume 5, p.1-412.

MEINERT, F. 1890. Philornis molesta, en paa Fugle snyltende Tachinarie. Vidensk. Medd. dansk naturh. Foren. 1:301317.

NIELSEN, J.C. 1913. On some South American species of the Genus Mydaea, parasitic on birds. Vidensk. Medd. dansk naturh. Foren. 65:251 256.

PAMPLONA, D.M. 1983. Sobre as espécies de Dasymorellia Malloch, 1923 (Diptera, Muscidae Muscinae). Bol. Mus. nac. Rio de J. (Zoologia) 305:1-10.

PAMPLONA, D.M. 1986. Sobre Morellia RobineauDesvoidy, 1830 neotropicais I: caracterização do gênero e descrição de três espécies novas (Diptera, Muscidae,Muscinae). Rev. bras. Biol. 46:651-665.

PONT, A.C. 1972. Family Muscidae. In A Catalogue of the Diptera of the Americas South of the United States, Museu de Zoologia, Universidade de São Paulo 97, p.1-111.

PONT, A.C. 1973. Studies on Australian Muscidae (Diptera). IV. A revision of the subfamilies Muscinae and Stomoxyinae. Austr. J. Zool., Suppl. 21:129-296. 
PONT, A.C. 1977. The Muscidae and Fanniidae (Insecta, Diptera) described by C.R.W. Wiedemann. Steenstrupia, 23:87-122.

PONT, A.C. 1981. The Linnaean species of the families Fanniidae, Anthomyiidae and Muscidae (Insecta: Diptera). Biol. J. Linnean Soc. 15:165-175.

PONT, A.C. 1984. A revision of the Fanniidae and Muscidae (Diptera) described by Fallén. Ent. Scandinavica 15: 277-297.

PONT, A.C. 1986a. A revision of the Fanniidae and Muscidae described by J.W. Meigen (Insecta: Diptera). Ann. naturh. Mus. Wien B 87:197-253.

PONT, A.C. 1986b. Studies on the Australian Muscidae (Diptera). VII. The Genus Atherigona Rondani. Aust. J. Zool., Suppl. Ser. 120:1-90.

PONT, A.C. 1997. The Muscidae and Fanniidae described by C.R.W. Wiedemann. Steenstrupia 23:87-122.

R0BINEAU DESVOIDY, A.J.B. 1830. Essai sur les Myodaires. Mémoires présentés par divers Savans à l’Académie Royales des Sciences 2:813 p.

RONDANI, C. 1856. Dipterologiae Italicae Prodromus. Vol. I. Genera Italica ordinis Dipterorum ordinatim disposita et distincta et in familias et stirpes aggregata. Stocchi, p.1-226.

RONDANI, C. 1866. Anthomyinae Italicae collectae distinctae et in ordinem dispositae. Atti Soc. ital. Sci. nat. 9:8217.

SCHINER, J.R. 1868. Diptera. In Reise der österreichischen Fregatte Novara um die Erde in den Jahren 1857, 1858, 1859, unter den Befehlen des Commodore B. von Wüllerstorf-Urbair. Zool. Theil, 2, 1 (B), p.1-388.

SCHNABL, J. 1911. Dipterologische Sammelreise nach Korsika. (Dipt.). Ausgeführt im Mai und Juni 1907 von Th. Becker, A. Kuntze, J. Schnabl und E. [sic] Villeneuve. (1. Fortsetzung.) Anthomyidae. Dtsch. ent. Z. 1911:62 100.

SCHNABL, J. \& DZIEDZICKI, H. 1911. Die Anthomyiden. Nova Acta Leop. Carol. 95:55 358.

SÉGUY, E. 1937. Diptera Fam. Muscidae. In Wytsman, P., Genera Insectorum. Fasc. 205, p.1-604.

SHANNON, R.C. \& DEL PONTE, E. 1926. Sinopsis parcial de los Muscoideos Argentinos. Rev. Inst. bact. Dep. Nac. Hig. B. Aires 4:549590.

SKIDMORE, P. 1985. The biology of the Muscidae of the world. Series Entomologica 29, p.1-550.

SNYDER, F.M. 1941. Contribution to a Revision of Neotropical Mydaeini (Diptera: Muscidae). Amer. Mus. Novit. 1134:1-22.

SNYDER, F.M. 1949a. Notes and Descriptions of some Phaoniinae (Diptera: Muscidae). Amer. Mus. Novit. 1402:1-25.

SNYDER, F.M. 1949b. New Genera and Species of Lispinae (Diptera, Muscidae). Amer. Mus. Novit. 1403:1-9.
SNYDER, F.M. 1949c. Revision of Neomuscina Townsend. Amer. Mus. Novit. 1404:1-39.

SNYDER, F.M. 1951. New Neotropical Muscidae (Diptera). Amer. Mus. Novit. 1494:1-11.

SNYDER, F.M. 1954. A Revision of Cyrtoneurina Giglio Tos, with Notes on Related Genera (Diptera, Muscidae). Bull. Amer. Mus. nat. Hist. 103:417 464.

SNYDER, F.M. 1957. Notes and Descriptions of some Neotropical Muscidae (Diptera). Bull. Amer. Mus. nat. Hist. 113:437490.

STEIN, P. 1904. Die Amerikanischen Anthomyiden des Königlichen Museums für Naturkunde zu Berlin und des Ungarischen National Museums zu Budapest. Ann. hist. nat. Mus. hung. 2:414 495.

STEIN, P. 1911. Die von Schnuse in Südamerika gefangenen Anthomyiden. Arch. für Naturgesch. 77:61 189.

STEIN, P. 1918. Zur weitern Kenntnis aussereuropäischer Anthomyiden. Ann. hist. nat. Mus. hung. 16:147 244.

STEIN, P. 1919. Die Anthomyidengattungen der Welt, analytisch bearbeitet, nebst einem kritisch systematischen Verzeichnis aller aussereuropäische Arten. Arch. für Naturgesch. 83 A 1:85 178.

THOMSON, C.G. 1869. Diptera. Species nova descripsit. In Kongliga svenska fregatten Eugenies Resa omkring jorden under befäl af C.A. Virgin, åren 1851-1853. Part 2, Zoologi, 1, Insecta: p.443 614.

TOWNSEND, C.H.T. 1892. Catalogue of the described South American Species of Calyptrate Muscidae. Ann. N. Y. Acad. Sci. 7:144.

TOWNSEND, C.H.T. 1915. Nine new tropical American genera de Muscoidea. Insecutor Inscitiae Menstruus 3:91 97.

TOWNSEND, C.H.T. 1916. Designations de Muscoid Genotypes, with New Genera e Species. Insec. Inscit. menst. 4:4 12.

TOWNSEND, C.H.T. 1919. New Genera e Species de Muscoid Flies. Proc. U. S. nat. Mus. 56:541 592.

TOWNSEND, C.H.T. 1927. Synopse dos gêneros muscoideos da região humida tropical da America, com gêneros e espécies novas. Rev. Mus paul. 15:203 385.

TOWNSEND, C.H.T. 1932. Five new Brazilian Oestromuscoid genera. Rev. Ent. 2:105 107.

WALKER, F. 1861. Characters of undescribed Diptera in the Collection of W. W. Saunders, Esq., F.R.S., \& c. [Part.]. Trans. ent. Soc. London 5:297 334.

WESTWOOD, J.O. 1840. Order XIII. Diptera Aristotle. (Antliata Fabricius. Halteriptera Clairv.), pp. 125-154. In An Introduction to the Modern classification of Insects; founded on the natural habits and corresponding organisation of the different families. Synopsis of the genera de British Insects, London, p.1-158. 
WIEDEMANN, C.R.W. 1824. Munus Rectoris in Academia Christiana Albertina aditurus Analecta entomologica ex Museo Regio Havniensi maxime congesta prdeert iconibusque illustrat., Kiliae, p.1-60.

WIEDEMANN, C.R.W. 1830. Aussereuropäische zweiflügelige Insekten. Part II, p.1-684.

WILLISTAON, S. W. 1896. On the Diptera of St. Vincent (West Indies). Trans. ent. Soc. London 1896:253 446.

WULP, F. M. van der. 1883. Amerikaansche Diptera. Tijdschr. Ent. 26:160.

WULP, F. M. van der. 1896a. Group Muscinae. [Part.] In Godman, F.D., \&O. Salvin [editors], BiologiaCentraliAmericana.ZoologiaInsecta-Diptera, 2 (part 129), p.291-304.

WULP, F. M. van der. 1896b. Group Anthomyiinae. [Part.] In Godman, F.D., \& O. Salvin [editors], Biologia Centrali Americana. Zoologia-Insecta-Diptera, 2 (part 132), p.321-344.

Título: Diptera Muscidae do Estado do Rio de Janeiro (Brasil)

Autores: Márcia Souto Couri \& Cláudio Jose Barros de Carvalho

Biota Neotropica, Vol. 5 ( número 2): 2005

http://www.biotaneotropica.org.br/v5n2/pt/ abstract?inventory+BN01505022005

Recebido em 14/12/04 - Revisado em: 01/05/05

Publicado em 03/08/2005

ISSN 1676-0603

http://www.biotaneotropica.org.br 